\title{
Biofunctionalization of silicone rubber with microgroove-patterned surface and carbon-ion implantation to enhance biocompatibility and reduce capsule formation
}

\author{
This article was published in the following Dove Press journal: \\ International Journal of Nanomedicine \\ 25 October 2016 \\ Number of times this article has been viewed
}

\section{Ze-yuan Lei \\ Ting Liu \\ Wei-juan Li \\ Xiao-hua Shi \\ Dong-li Fan}

Department of Plastic and Cosmetic Surgery, XinQiao Hospital, The Third Military Medical University, ChongQing, People's Republic of China
Correspondence: Dong-li Fan Department of Plastic and Cosmetic Surgery, XinQiao Hospital, The Third Military Medical University, ChongQing 400037, People's Republic of China

Tel +862368763288

Email dlfan46I2@I63.com
Purpose: Silicone rubber implants have been widely used to repair soft tissue defects and deformities. However, poor biocompatibility can elicit capsule formation, usually resulting in prosthesis contracture and displacement in long-term usage. To overcome this problem, this study investigated the properties of silicone rubber materials with or without a microgroovepatterned surface and with or without carbon (C)-ion implantation.

Materials and methods: Atomic force microscopy, X-ray photoelectron spectroscopy, and a water contact angle test were used to characterize surface morphology and physicochemical properties. Cytocompatibility was investigated by a cell adhesion experiment, immunofluorescence staining, a Cell Counting Kit-8 assay, and scanning electron microscopy in vitro. Histocompatibility was evaluated by studying the inflammatory response and fiber capsule formation that developed after subcutaneous implantation in rats for 7 days, 15 days, and 30 days in vivo.

Results: Parallel microgrooves were found on the surfaces of patterned silicone rubber (P-SR) and patterned C-ion-implanted silicone rubber (PC-SR). Irregular larger peaks and deeper valleys were present on the surface of silicone rubber implanted with $\mathrm{C}$ ions (C-SR). The silicone rubber surfaces with microgroove patterns had stable physical and chemical properties and exhibited moderate hydrophobicity. PC-SR exhibited moderately increased dermal fibroblast cell adhesion and growth, and its surface microstructure promoted orderly cell growth. Histocompatibility experiments on animals showed that both the anti-inflammatory and antifibrosis properties of PC-SR were slightly better than those of the other materials, and there was also a lower capsular contracture rate and less collagen deposition around implants made from PC-SR.

Conclusion: Although the surface chemical properties, dermal fibroblast cell growth, and cell adhesion were not changed by microgroove pattern modification, a more orderly cell arrangement was obtained, leading to enhanced biocompatibility and reduced capsule formation. Thus, this approach to the modification of silicone rubber, in combination with $\mathrm{C}$-ion implantation, should be considered for further investigation and application.

Keywords: silicone rubber, biocompatibility, capsule formation, microgroove, C-ion implantation

\section{Introduction}

The use of biological materials and soft tissue substitute implants in surgical repair is the main method of treatment for soft tissue defects and deformities. For use in plastic surgery, a bioimplant material should ideally have high tear strength, low hardness, high thermal stability, high chemical resistance, and good biocompatibility. ${ }^{1}$ Silicone rubber and silicone rubber-based materials are the biomaterials most commonly used in 
clinical implants. This has been the case for many years, but there is increasing evidence suggesting that the intrinsically hydrophobic nature of silicone rubber surface leads to poor cell adhesion and tissue compatibility between the implant and surrounding tissues, resulting in capsule formation and to gradual thickening and contracture of these tissues. ${ }^{2}$ In addition, these capsular voids also encourage bacterial infection and invasion as well as inflammation during longterm use. ${ }^{3}$ Although silicone rubber implants are bioinert and workable, they have been involved in a great number of adverse reactions, sometimes occurring decades after implantation, and to date no satisfactory solution to the problems of fibrosis and capsule formation has been found.

A possible solution is represented by modification of the silicone rubber surface to minimize hydrophobic interaction and improve cell adhesion. In recent years, a large number of studies supporting the use of surface modification to reduce bacterial adhesion and improve the biocompatibility of silicone rubber have been reported. A variety of surface modification methods, such as coating with carbon nanotubes, plasma spraying, sintering, and electrochemical deposition, can reduce the surface hydrophobicity of silicone rubber, increase the adhesion and proliferation of fibroblasts, and significantly improve cytocompatibility. ${ }^{4-7}$ Liu et $\mathrm{al}^{8}$ showed that surface modification with zwitterionic polymers could remarkably improve the wettability of a silicone rubber surface and provide excellent resistance to platelet adhesion, thereby significantly enhancing blood compatibility.

Our previous studies showed that remodeling of a silicone rubber surface by $\mathrm{C}$-ion implantation could effectively improve cytocompatibility. This improvement was attributed to changes in surface characteristics, including surface chemistry, surface roughness, and wettability. ${ }^{9,10}$ The C-ion implantation also changed the surface morphology of the silicone rubber, but whether such changes in surface topography have any important effects on its functions as a biomaterial, and in particular its cytocompatibility, needs further investigation. With this aim in mind, in this study, silicone rubber surfaces are modified by the imposition of a novel microgroove pattern and by $\mathrm{C}$-ion implantation. The surface physical and chemical properties of the modified materials are determined. A series of in vitro and in vivo experiments are conducted to analyze and evaluate the biocompatibility of the silicone rubber with and without surface modification. In addition, the effect on capsule formation when the modified materials are used as implants is investigated, with the hope of developing a soft tissue filling material that meets clinical needs with relatively few side effects.

\section{Materials and methods}

\section{Sample preparation}

The Twenty-sixth Research Institute of China Electronic Technology Group Corporation was responsible for providing samples of $12 \times 12 \mathrm{~cm}$ square glass masters with microgroove. Equal amounts (3 $\mathrm{mL})$ of clinical-quality liquid silicone rubber components SR-A and SR-B (provided by Chenguang Research Institute of Chemical Engineering, People's Republic of China) were mixed in a 1:1 ratio and poured on to a metal mold plate (to produce SR samples) or on to a glass microgrooved master (to produce P-SR samples). Polymerization to produce a silicone rubber membrane took 3-4 hours at room temperature. Furthermore, the surfaces of some SR and P-SR samples were then modified by $\mathrm{C}$-ion implantation at a dose of $1 \times 10^{16} \mathrm{ions} / \mathrm{cm}^{2}$ to produce C-SR and PC-SR samples, respectively. All operations were carried out under sterile conditions, and all the experiments in this study were divided into the following four groups: 1) SR group (silicone rubber with unmodified smooth surface); 2) P-SR group (silicone rubber with surface modified by microgrooves); 3 ) C-SR group (silicone rubber with surface modified by C-ion implantation); and 4) PC-SR group (silicone rubber with surface modified by both microgrooves and C-ion implantation).

\section{Micromorphological observation and physicochemical analysis}

All samples (SR, P-SR, C-SR, and PC-SR), in the form of $1 \times 1 \mathrm{~cm}$ squares, were cleaned ultrasonically $(20 \mathrm{~W}$, 2 minutes). After drying and cooling, the surface morphology of the materials was observed by atomic force microscopy (SAP 400; Seiko Instrument Inc., Chiba, Japan). A NanoNavi analysis system was used to measure the microgroove parameters of the P-SR and PC-SR samples. The physicochemical properties of all the sample surfaces were evaluated and characterized by X-ray photoelectron spectroscopy (ESCALAB 250; Thermo Fisher Scientific, Waltham, MA, USA), and the water contact angle was measured with a drop shape analysis system (DSA100; Kruss, Hamburg, Germany).

\section{Cell culture}

Human dermal fibroblast cell lines were obtained from the Cell Bank of Chinese Academy of Sciences (Shanghai, People's Republic of China) and cultured in Dulbecco's Modified Eagle's Medium (Thermo Fisher Scientific), containing 10\% fetal bovine serum (FBS) (Thermo Fisher Scientific), penicillin (100 U/mL) (Sigma-Aldrich Co., 
St Louis, MO, USA), and streptomycin $(100 \mu \mathrm{g} / \mathrm{mL})$ (SigmaAldrich Co.) in a $5 \% \mathrm{CO}_{2}$ incubator at $37^{\circ} \mathrm{C}$.

\section{Cytocompatibility experiment}

All samples were cut into the appropriate sizes to fit into the bottoms of the wells in 6-, 24-, and 96-well plates. Cells were collected and seeded into the plates at different densities per well and left overnight. A cell adhesion assay was performed as described previously. ${ }^{9}$ The cell adhesion rate (\%) was calculated as (number of adherent/number of seeded cells) $\times 100 \%$. Cell viability was assessed by a Cell Counting Kit-8 (CCK-8) assay (Dojindo, Kumamoto, Japan) at 1 day, 2 days, 4 days, and 6 days, according to the manufacturer's protocol.

Cell morphology was observed by scanning electron microscope (AMRAY 1000-B; Amray Inc., Bedford, MA, USA). The dermal fibroblasts cytoskeleton was observed by a laser scanning confocal microscope and immunofluorescence staining with fluorescein isothiocyanate-labeled actin Tracker probes (Beyotime, Shanghai, People's Republic of China). A Western blot assay (performed as described previously ${ }^{9}$ ) was used to detect the expression of talin, zyxin, and vinculin proteins, which are involved in the process of cell adhesion to various material surfaces. The primary antibodies used in this study were as follows: rabbit anti-talin (1:1,000; EMD Millipore, Billerica, MA, USA), rabbit anti-zyxin (1:1,000; Epitomics, Burlingame, CA, USA), and rabbit anti-vinculin (1:500, Sigma-Aldrich Co.). The secondary antibody was goat anti-rabbit $\operatorname{IgG}$ horseradish peroxidase $(1: 4,000$; Sigma-Aldrich Co.).

\section{Animal study}

All samples were cut into $1 \times 1 \mathrm{~cm}$ squares and disinfected. Sixteen Sprague Dawley adult rats (female, body weight $=160-200 \mathrm{~g}$ ) were disposed with $3 \%$ pentobarbital sodium (Sigma-Aldrich Co.). After anesthetizing, four parallel incisions $(1 \mathrm{~cm})$ were then performed in each subject. All experimental samples were implanted subcutaneously along the back region, and the incisions were sutured with 1-0 sutures. The animals were sacrificed 7 days, 15 days, and 30 days after operation, and the tissues surrounding the implants were collected as specimens to explore the histocompatibility of the implanted samples. There were four duplicate determinations for each group. At the each point after implantation, hematoxylin and eosin staining was used to observe changes in inflammatory cell infiltration around the implants. The fiber texture in the tissue around the implants was revealed by Masson staining and immunohistochemistry with the following primary antibodies: rabbit anti- $\alpha$-smooth muscle actin (anti- $\alpha$-SMA) (1:100; Santa Cruz Biotechnology Inc., Dallas, CA, USA) and rabbit anti-vimentin (1:100; Abcam, Cambridge, UK). In particular, this study was approved by the Animal Care and Ethics Committee at the Third Military Medical University (Chongqing, People's Republic of China), and all procedures were conducted in compliance with approved institutional animal care and protocols.

\section{Statistical analysis}

The data were expressed as mean $\pm \mathrm{SD}$, and all statistical analyses were performed using the SPSS 19.0 statistical software package (IBM Corporation, Armonk, NY, USA). Statistical difference was determined by one-way analysis of variance with multiple comparisons and Student's $t$-test. In all statistical evaluations, $P<0.05$ was considered as statistically significant.

\section{Results \\ Morphologic observation}

SR, P-SR, C-SR, and PC-SR were produced as described. The atomic force microscopy results shown in Figure 1A and B and summarized in Table 1 reveal that the SR had a smooth surface and that the microgrooves on the surface of the P-SR were uniformly distributed. After C-ion implantation, the surface of the C-SR had irregular larger peaks and deep valleys, while the surface of the PC-SR exhibited a concave-convex topography in the uniformly distributed microgrooves. The results from the NanoNavi analysis measurements of the microgroove parameters indicate that the length of each microgroove is $\sim 6 \mu \mathrm{m}$ (Figure 1C).

\section{Surface properties}

The X-ray photoelectron spectroscopy results indicate that the chemical compositions ( $\mathrm{C}, \mathrm{O}, \mathrm{Si})$ of SR and P-SR were almost identical to one another, as were those of C-SR and PC-SR with the latter two groups showing higher proportions of $\mathrm{C}$ (Figure 2A and B). These results suggest that surface modification with microgrooves did not change the proportion of chemical elements in the silicone rubber but that $\mathrm{C}$-ion implantation did lead to such changes. In addition, water contact angle test was used to judge the change of surface wettability and the hydrophobic property. As shown in Figure $2 \mathrm{C}$, the data of water contact angle increased in the order $\mathrm{C}$-SR $<\mathrm{SR}<\mathrm{PC}-\mathrm{SR}<\mathrm{P}$-SR, indicating that the microgroove structure significantly increased the hydrophobicity of the silicone rubber surface, while C-ion implantation increased its hydrophilicity. 
A
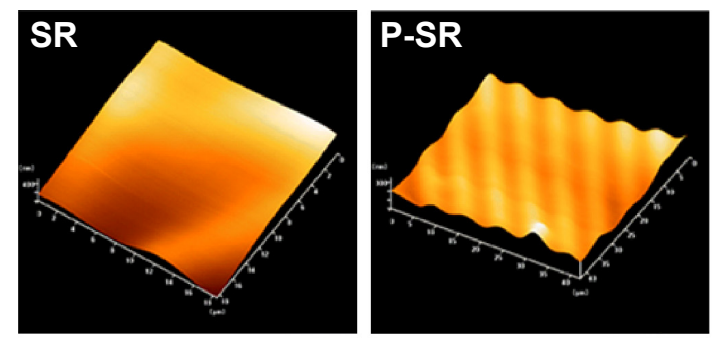

B
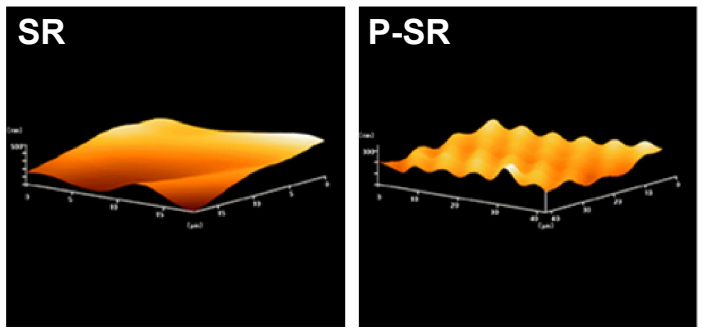
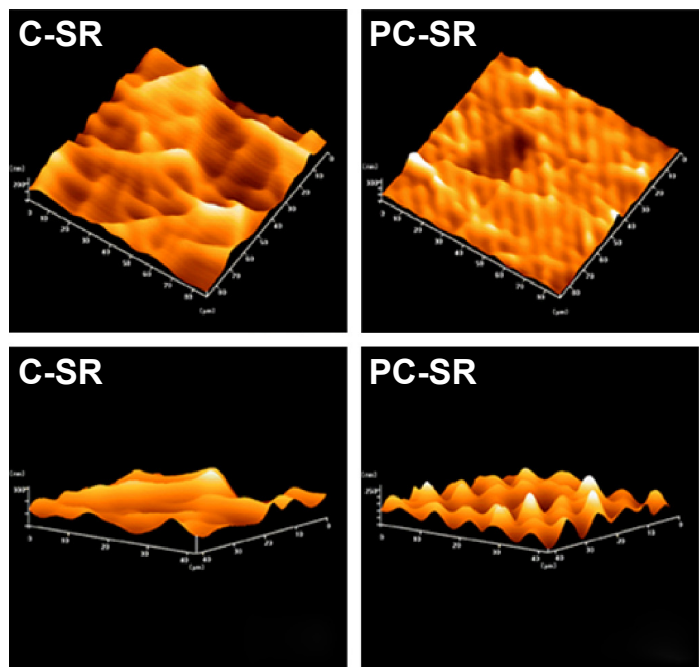

C

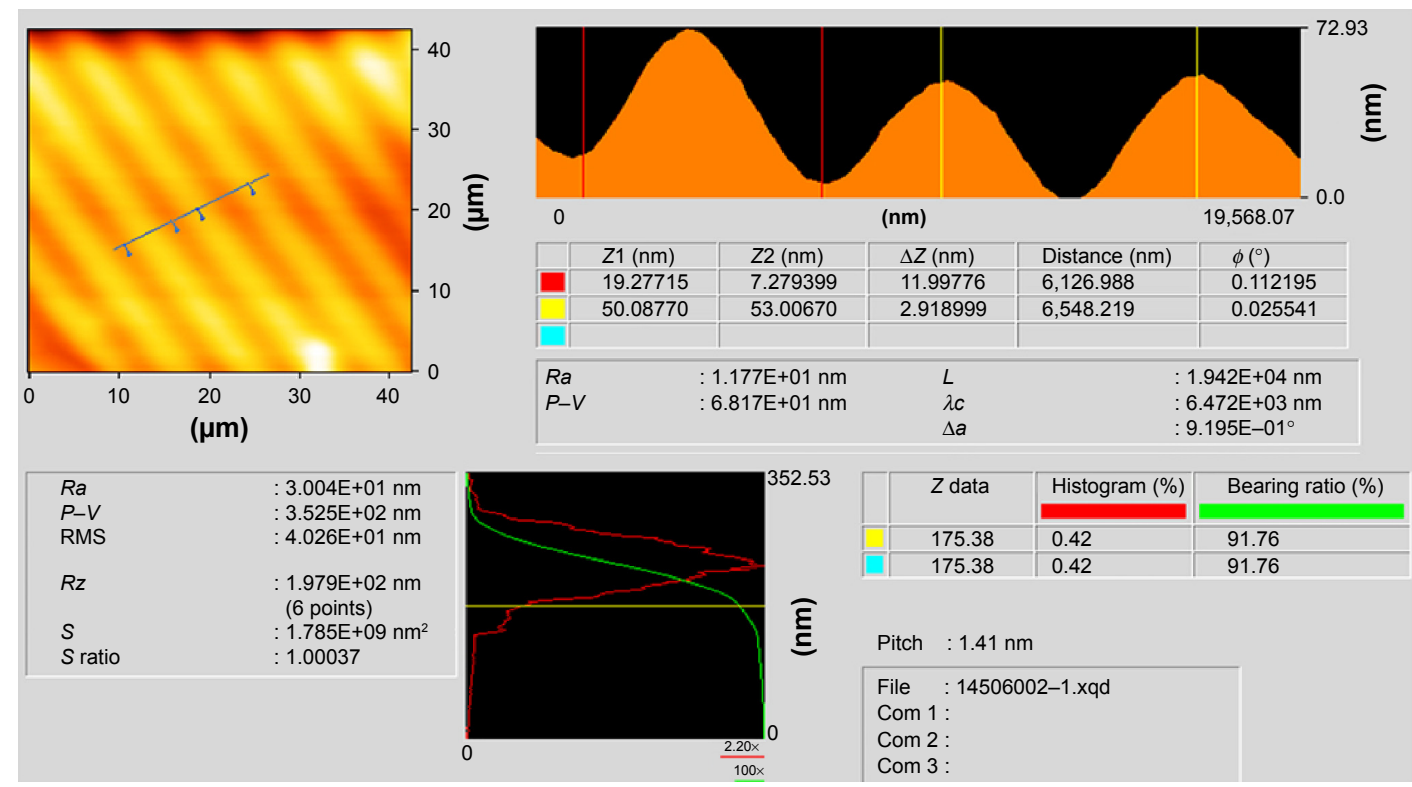

Figure I Surface topography of samples as observed by AFM in each group.

Notes: (A) Front view. (B) Side view. (C) Surface microgroove parameters.

Abbreviations: SR, silicone rubber; P-SR, patterned silicone rubber; C-SR, C-ion-implanted silicone rubber; PC-SR, patterned C-ion-implanted silicone rubber; RMS, root mean square.

\section{Cytocompatibility analysis}

Previous studies have found that changes in the physical and chemical properties of a biomaterial can regulate the reaction between the material and the body, with significant impacts

Table I Surface topography for each group

\begin{tabular}{|c|c|c|c|}
\hline Group & $\begin{array}{l}\text { Microgroove } \\
\text { modification }\end{array}$ & C-ion & Surface topography \\
\hline$\overline{S R}$ & No & No & Smooth \\
\hline P-SR & Yes & No & Parallel microgrooves \\
\hline C-SR & No & Yes & $\begin{array}{l}\text { Irregular larger peaks and deeper } \\
\text { valleys }\end{array}$ \\
\hline PC-SR & Yes & Yes & $\begin{array}{l}\text { Irregular concave-convex structure } \\
\text { based on parallel microgrooves }\end{array}$ \\
\hline
\end{tabular}

Abbreviations: SR, silicone rubber; P-SR, patterned silicone rubber; C-SR, C-ionimplanted silicone rubber; PC-SR, patterned C-ion-implanted silicone rubber. on cell behavior, including cell proliferation, migration, and differentiation capacity. Laser confocal microscope observations of the dermal fibroblasts skeleton (Figure 3A) showed a disordered arrangement of dermal fibroblasts around SR and C-SR, while cell growth around P-SR was similar to that around SR, but with an orderly arrangement of cells. There was an increased area of cell growth around C-SR. The cell arrangement around PC-SR was more orderly compared with that around C-SR, as confirmed by the scanning electron microscope images (Figure 3B).

The results of Cell Counting Kit-8 analysis (Figure 3C) showed significantly increased cell growth around C-SR and PC-SR compared with SR and P-SR, while the difference between C-SR and PC-SR was not statistically significant. 

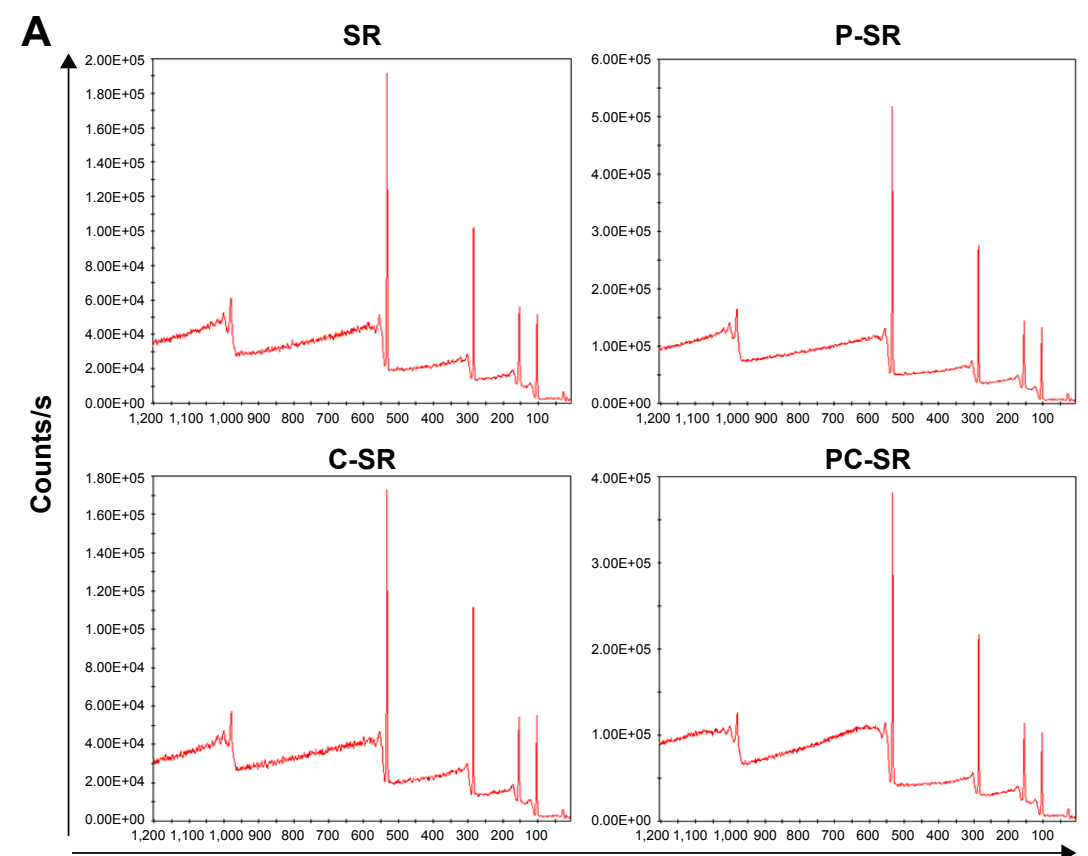

B

\begin{tabular}{|l|l|l|l|}
\hline Group & Si 2p & C 1s & 0 1s \\
\hline SR & 30.58 & 40.13 & 29.30 \\
\hline P-SR & 30.89 & 39.59 & 29.52 \\
\hline C-SR & 29.35 & 45.22 & 25.43 \\
\hline PC-SR & 30.08 & 42.14 & 27.78 \\
\hline
\end{tabular}

Binding energy $(\mathrm{eV})$

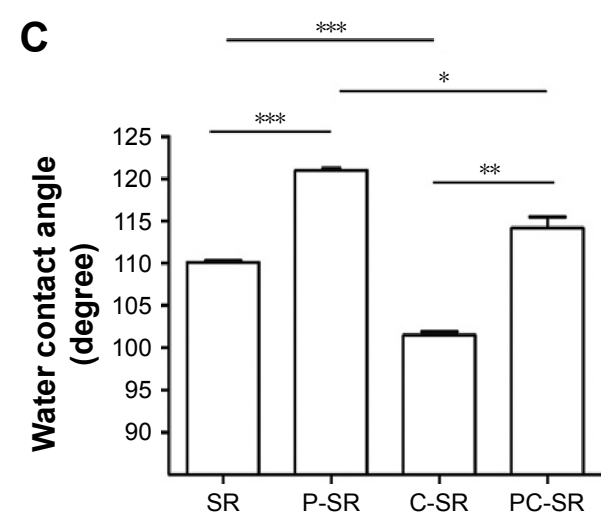

Figure 2 Surface physical and chemical properties for each group of samples.

Notes: (A, B) XPS analysis of the chemical composition of SR, P-SR, C-SR, and PC-SR. (C) Water contact angle of SR, P-SR, C-SR, and PC-SR ( $* P<0.05$, $* * P<0.01$, $* * * P<0.00$ I)

Abbreviations: SR, silicone rubber; P-SR, patterned silicone rubber; C-SR, C-ion-implanted silicone rubber; PC-SR, patterned C-ion-implanted silicone rubber; s, seconds.

Similar results were found from the cell adhesion experiments. The cell adhesion rate was significantly increased for C-SR and PC-SR compared with SR and P-SR, respectively (Figure 3D). Furthermore, as shown in Figure 3E and F, Western blot results displayed that protein expression for the silicone rubber surfaces modified by $\mathrm{C}$-ion implantation was better than for the surfaces not modified in this way and that protein expression for C-SR and PC-SR was consistent with the cell adhesion results. Together, these results indicate that $\mathrm{C}$-ion implantation had significant effects on cell growth and cell adhesion and that the surface morphology could be altered to encourage a more orderly arrangement of fibroblasts, possibly leading to improved cytocompatibility.

\section{Effect of microgroove surface pattern on local inflammatory reaction}

The chronic inflammatory reaction is an important index of the histocompatibility of biomaterial implants. In this study, the results of hematoxylin and eosin staining show that in the acute stage following implantation (7 days; Figure 4A), there was no statistically significant inflammatory cell infiltration or changes in cell numbers with any of the silicone rubber materials. In the subacute period (15 days; Figure 4B) and the chronic period (30 days; Figure 4C), the number of inflammatory cells around the implants decreased gradually and there was clearly less inflammatory reaction around the implants with C-ion implantation (C-SR and PC-SR) than around those without (SR and P-SR). In addition, there were lower numbers of inflammatory cells around the implants with a microgroove surface pattern (P-SR and PC-SR) than around those without (SR and C-SR). Together, these results indicate that silicone rubber with $\mathrm{C}$-ion implantation has a certain degree of anti-inflammatory activity, as does silicone rubber with a microgroove surface pattern.

\section{Effects of microgroove surface pattern on collagen deposition and inhibition of fibrous capsule formation}

The thickness of the fibrous capsule formed around an implant is another important index for histological evaluation of biomaterials. In this study, we observed the formation of the capsule around the implant material by Masson staining. The results show that 7 days after implantation (Figure 5A), there were no significant differences in expression of collagen among the groups. After 15 days of implantation (Figure 5B), increased staining was observed for all materials. However, there was greater collagen expression for P-SR than for SR, while there was no significant difference between collagen expression for PC-SR and that for C-SR. After 30 days of implantation (Figure 5C), collagen expression for PC-SR was significantly less than that for C-SR, and collagen bundles exhibited a loose storiform arrangement (Figure 5C). 

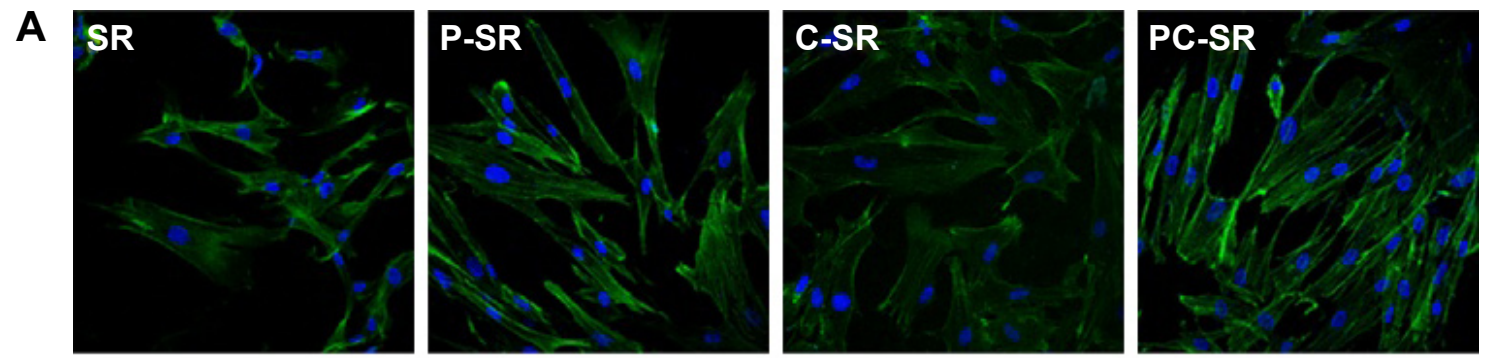

B
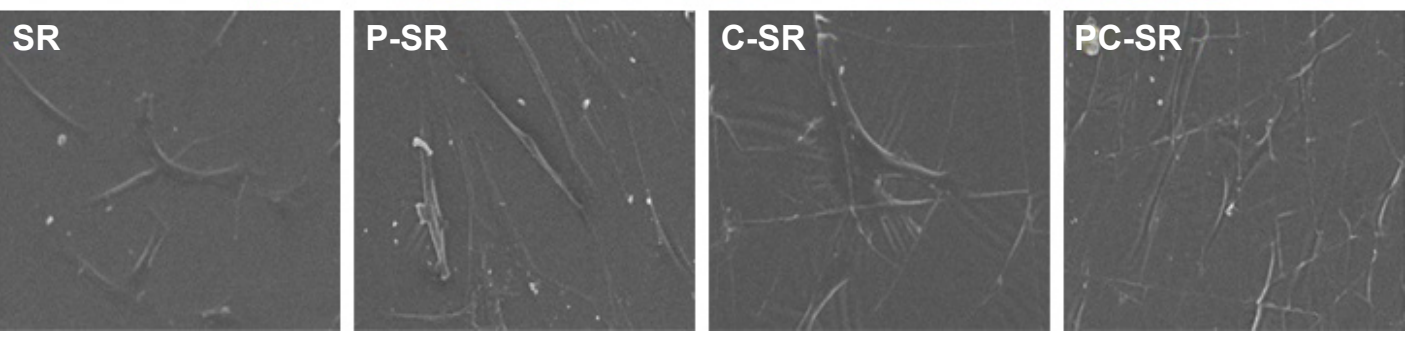

C

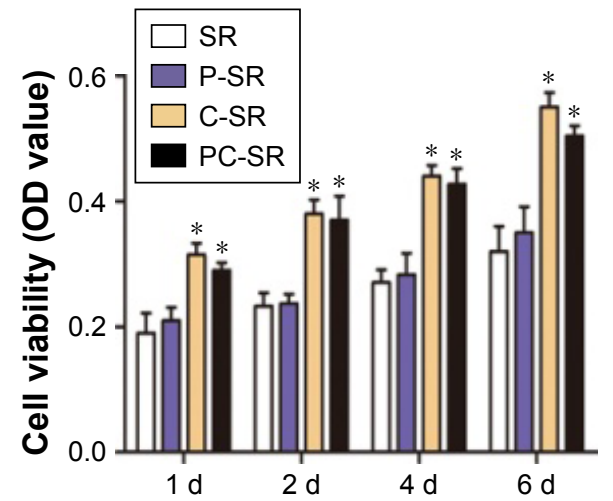

D

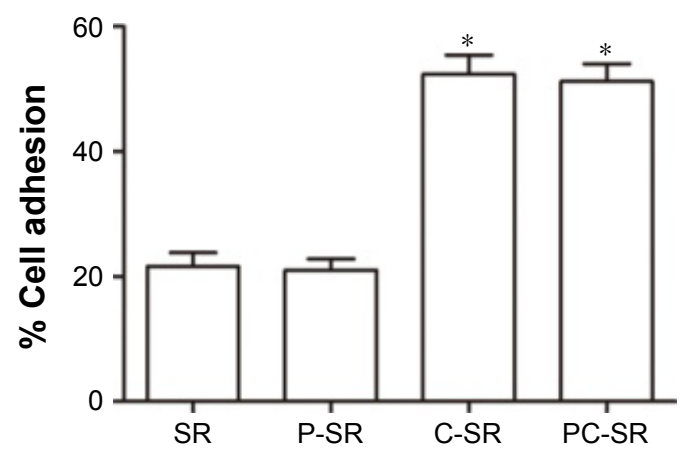

E

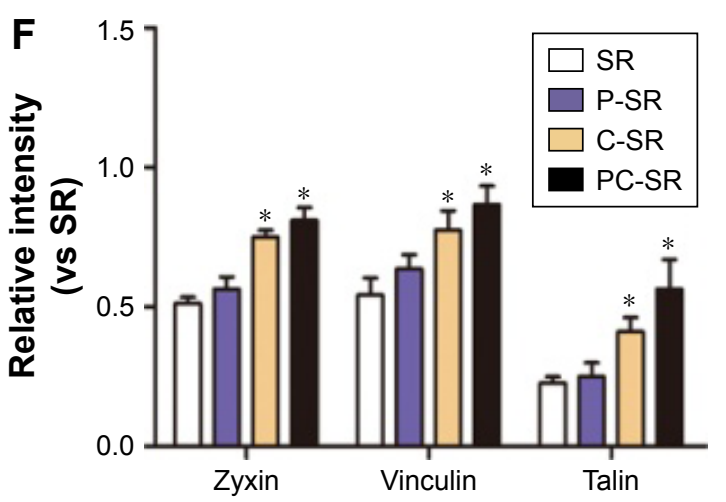

Figure 3 Analysis of cytocompatibility in the different groups.

Notes: (A) Immunofluorescence microscope observations of fibroblast cytoskeleton using FITC-labeled actin tracer. (B) SEM observations of the arrangement of cells. (C) Cell viability as analyzed by CCK-8 assay $(* P<0.05)$. (D) Cell adhesion on different substrates $(* P<0.05)$. (E, $\mathbf{F})$ Western blot results showing the expressions of talin-I, vinculin, and zyxin in dermal fibroblasts cultured on SR, P-SR, C-SR, and PC-SR coated 6-well plates $(* P<0.05)$.

Abbreviations: SR, silicone rubber; P-SR, patterned silicone rubber; C-SR, C-ion-implanted silicone rubber; PC-SR, patterned C-ion-implanted silicone rubber; OD, optical density; d, days.

These data suggest that the silicone rubber surface modified with both $\mathrm{C}$-ion implantation and a microgroove pattern led to the generation of thinner and weaker tissue capsules and had better biocompatibility compared with the other surfaces.

Previous studies have suggested that $\alpha$-SMA and vimentin have important roles in the evaluation of tissue compatibility. The results of this study show that, 7 days after implantation of silicone rubber materials, the expressions of $\alpha$-SMA and vimentin were lower in each group, with no obvious difference among groups (Figures 6A and 7A). After 15 days and 30 days of implantation, the degree of expression of $\alpha$-SMA and vimentin increased gradually in each group (Figures 6B and $\mathrm{C}$ and $7 \mathrm{~B}$ and $\mathrm{C}$ ). However, the amount of fibrosis was clearly lower around the implants with C-ion implantation (C-SR and PC-SR) than around those without (SR and P-SR) at the same period. There was 
A

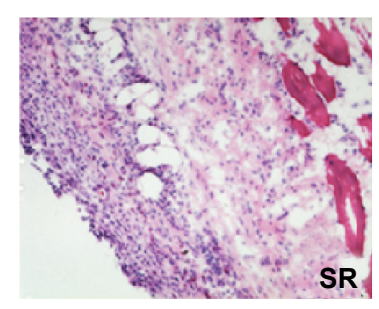

B

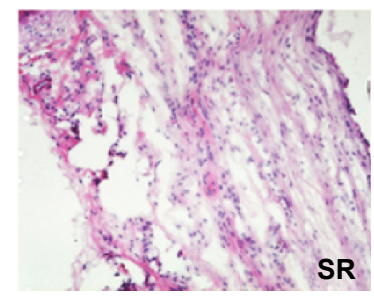

C

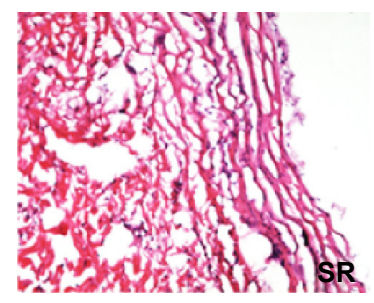

7 days
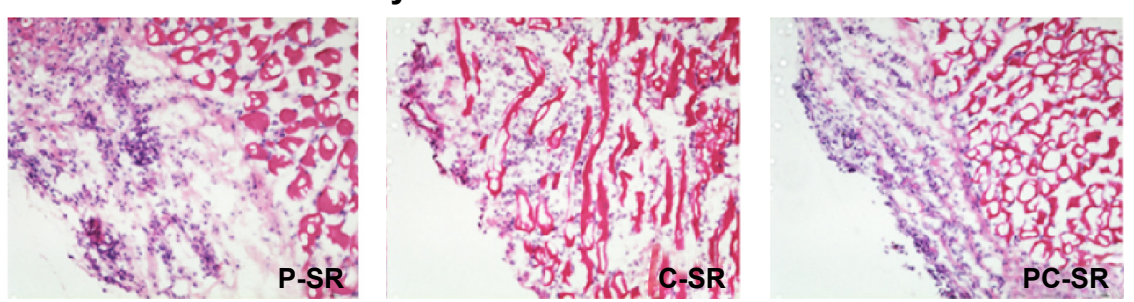

15 days
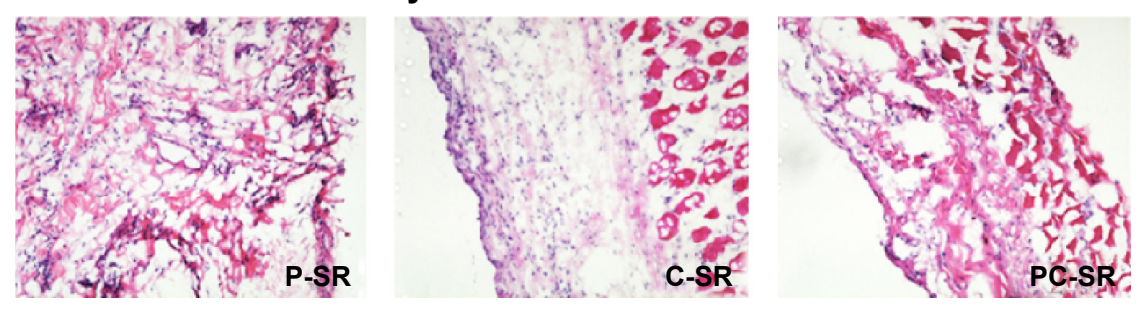

30 days
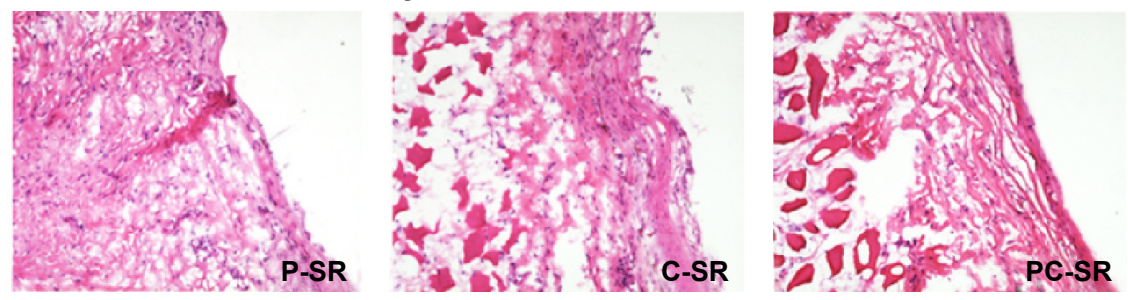

Figure $4 \mathrm{H} \& \mathrm{E}$ staining evaluating the local inflammatory reaction following implantation of silicone rubber materials. $(\times 200)$ Notes: (A) 7 days. (B) 15 days. (C) 30 days.

Abbreviations: SR, silicone rubber; P-SR, patterned silicone rubber; C-SR, C-ion-implanted silicone rubber; PC-SR, patterned C-ion-implanted silicone rubber.

A

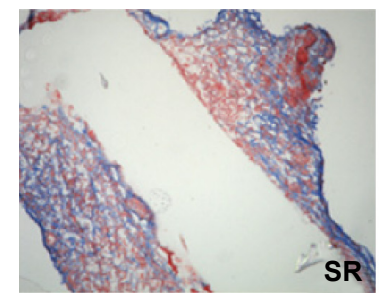

B

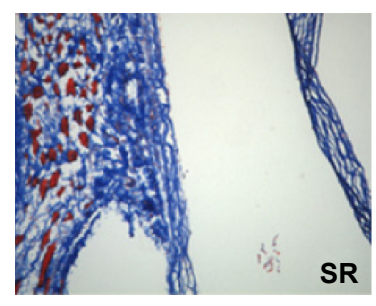

C

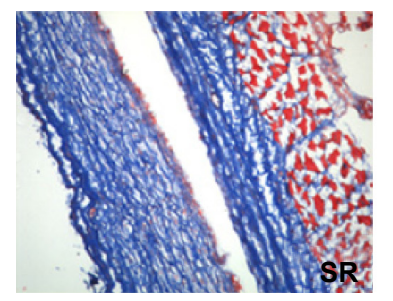

7 days
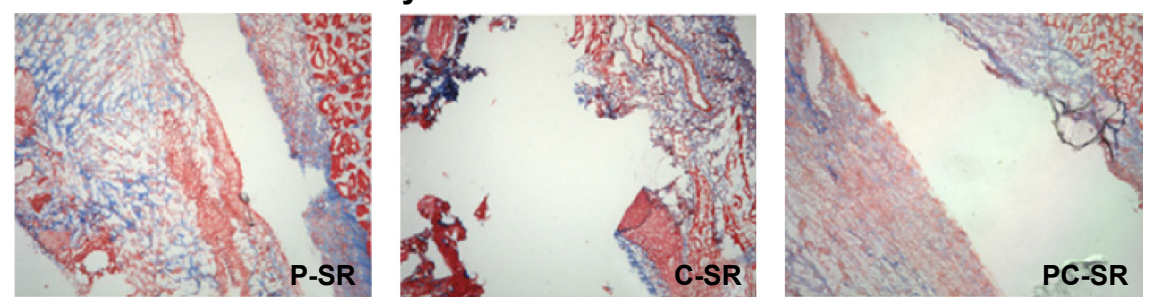

15 days
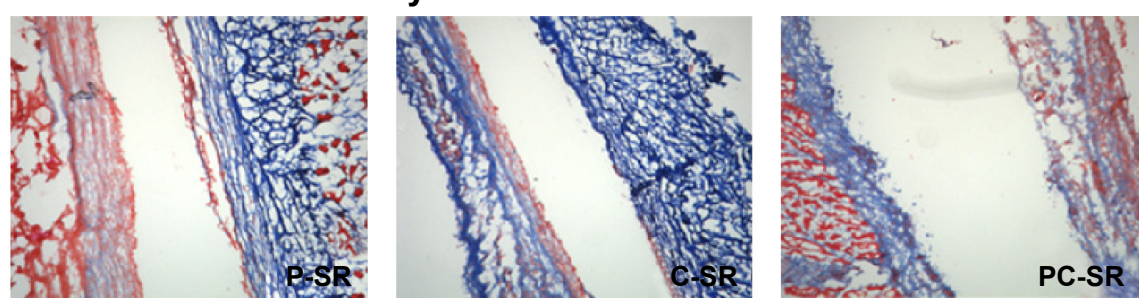

30 days
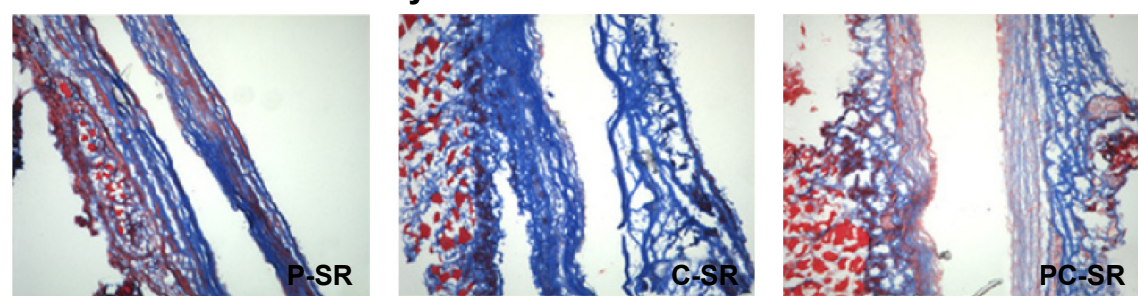

Figure 5 Masson staining evaluating the capsule thickness following implantation of silicone rubber materials. $(\times 200)$

Notes: (A) 7 days. (B) 15 days. (C) 30 days.

Abbreviations: SR, silicone rubber; P-SR, patterned silicone rubber; C-SR, C-ion-implanted silicone rubber; PC-SR, patterned C-ion-implanted silicone rubber. 
A

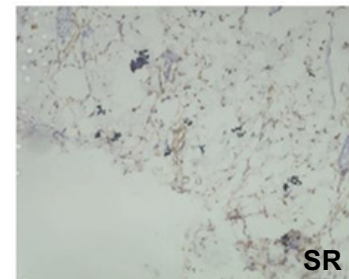

B

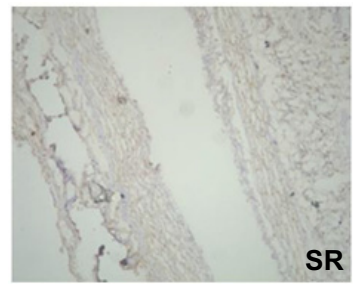

C

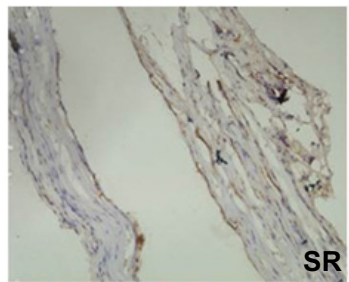

7 days

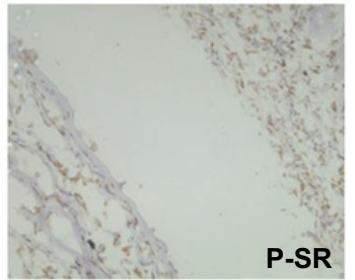

15 days

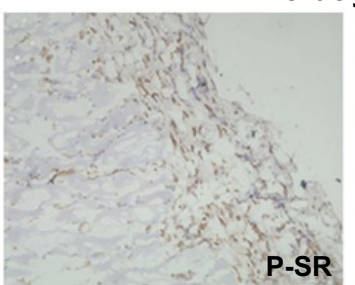

C-SR

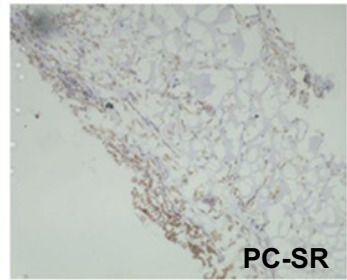

PC-SR

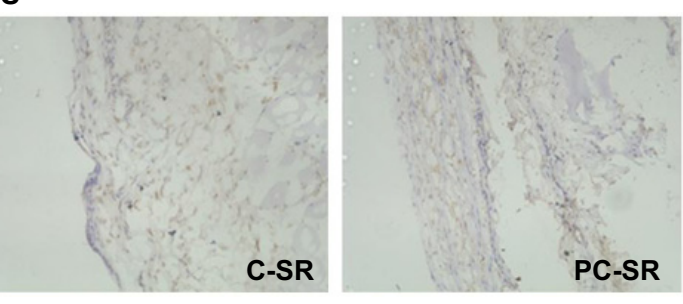

30 days
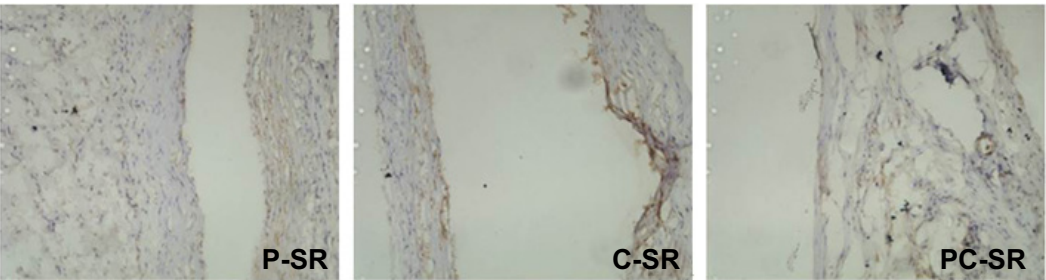

Figure 6 Positive expression degree of vimentin as analyzed by immunohistochemistry after implantation of silicone rubber materials. $(\times 200)$ Notes: (A) 7 days. (B) 15 days. (C) 30 days.

Abbreviations: SR, silicone rubber; P-SR, patterned silicone rubber; C-SR, C-ion-implanted silicone rubber; PC-SR, patterned C-ion-implanted silicone rubber.

A

SR

B

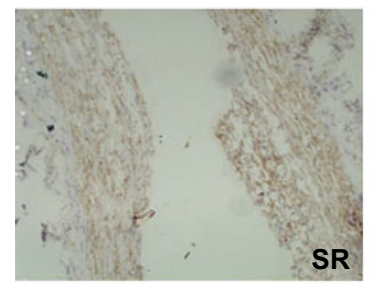

C

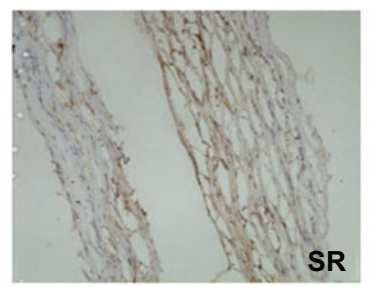

7 days
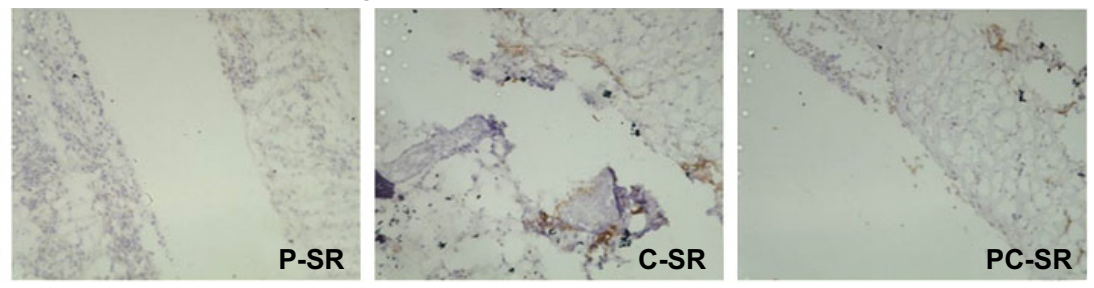

15 days
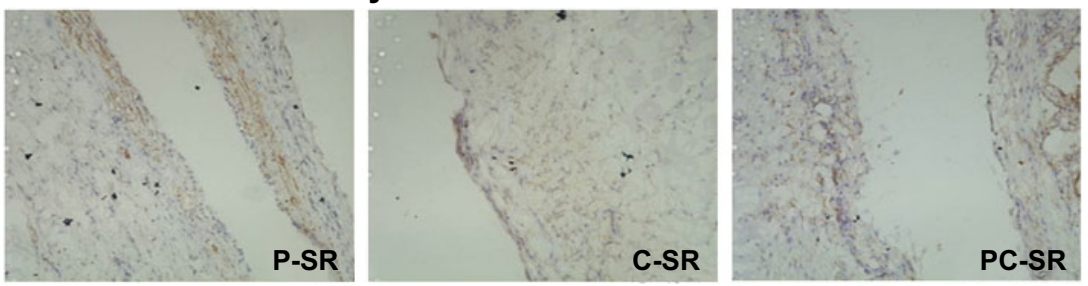

30 days
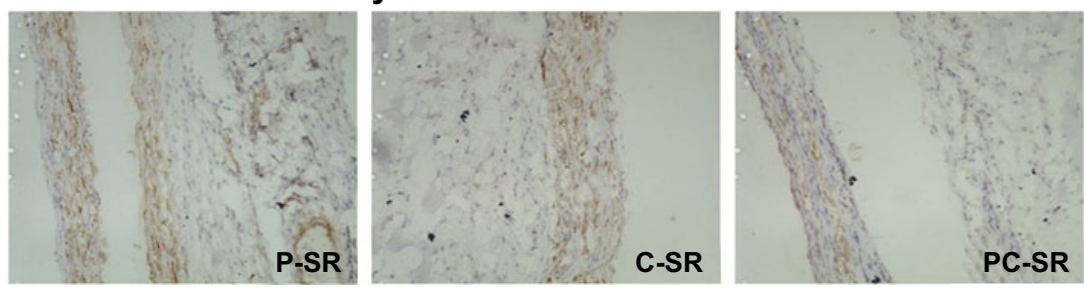

Figure 7 Positive expression degree of $\alpha$-SMA as analyzed by immunohistochemistry after implantation of silicone rubber materials. $(\times 200)$ Notes: (A) 7 days. (B) 15 days. (C) 30 days.

Abbreviations: SR, silicone rubber; P-SR, patterned silicone rubber; C-SR, C-ion-implanted silicone rubber; PC-SR, patterned C-ion-implanted silicone rubber. 
also less fibrosis around the implants with microgroove surface pattern (P-SR and PC-SR) than around those without (SR and C-SR). Together, these results suggest that the silicone rubber surfaces with $\mathrm{C}$-ion implantation exhibited a certain amount of antifibrosis activity, as did those with microgroove patterns.

\section{Discussion}

With the development of tissue engineering and regenerative medicine, more stringent requirements have been imposed on biological materials. In addition to appropriate physical and chemical properties, nontoxicity, and low production cost, of great importance among these requirements is biological compatibility. The most important method to improve the biocompatibility of such materials is alteration of their surface properties to control their reaction with the patient's body. ${ }^{11}$ The chemical, structural, and morphological properties of implanted materials can induce different biocompatibility responses. ${ }^{12-14}$ Previous studies have found that the surface topography of an implanted material is of great importance for morphogenesis as well as affecting the biological behavior of cultured cells such as cell proliferation, migration, and differentiation. ${ }^{15,16}$

Surface topography mainly involves grooves, ridges, ladders, and holes. The effects on cell compatibility of different patterns and feature sizes at the micrometer, submicrometer, and nanometer scales are different. ${ }^{17-19}$ Therefore, through the preparation of implantable materials with specific surface topography and chemical structure, it has been possible to regulate a series of functions involving cell proliferation, migration, and differentiation, as well as antibacterial properties, all of which contribute to improving biological compatibility and function of implant materials. In this context, we have succeeded in modifying the surface of silicone rubber, by imposition of a microgroove pattern and by changing the surface $\mathrm{C}$-ion content and distribution. Remarkably, our study demonstrated that the microgroove pattern did not alter the silicone rubber chemical composition, but the water contact angle was increased, suggesting that the hydrophobic properties of the surface were improved. With $\mathrm{C}$-ion implantation, the percentage of $\mathrm{C}$ on the surface of the material was increased significantly, and the water contact angle was decreased, improving the surface wettability of the material, thus suggesting that $\mathrm{C}$-ion implantation can enhance surface hydrophilicity.

Surface modification of silicone rubber by micropatterning or C-ion implantation could affect the biological behavior of cells. ${ }^{20,21}$ Tsuji et al ${ }^{22,23}$ found that a better adhesion of rat mesenchymal stem cells could be obtained by C-ion implantation at a dose of $3 \times 10^{15}$ ions $/ \mathrm{cm}^{2}$ on the surface of silicone rubber and that when this was done using raster graphics, cell growth could be induced along the direction of the pattern. In this study, we found that dermal fibroblasts grow faster and in a more orderly manner in the case of a silicone rubber surface modified with both microgrooves and C-ion implantation; cell adhesion rate was also increased significantly, and there was an increase in the amount of cell adhesion plaque proteins. This suggests that $\mathrm{C}$-ion implantation promoted cell proliferation. while the microgroove pattern facilitated more orderly cell growth. Thus, the combination of microgroove patterning and $\mathrm{C}$-ion implantation should significantly improve the cytocompatibility of silicone rubber.

Capsule contracture is considered to be an inevitable complication of the use of silicone rubber implants. ${ }^{24} \mathrm{~A}$ clinical study showed that the use of a silicone rubber prosthesis with a textured surface could reduce the incidence of capsular contracture compared with a smooth surface. ${ }^{25}$ In our study, P-SR showed weaker inflammatory stimulation, less collagen deposition, and thinner capsule thickness in vivo compared with SR. Positive expression of $\alpha$-SMA and vimentin reflects the degree of collagen deposition, which is used to evaluate the histocompatibility of implant materials. High levels of $\alpha$-SMA and vimentin usually lead to capsular contracture. ${ }^{26} \mathrm{We}$ found that $\alpha$-SMA and vimentin expression gradually increased with time for all implant materials studied, but the expression with PC-SR was lower than with the other materials. It appears that the combination of $\mathrm{C}$-ion implantation and surface microgroove patterning of silicone rubber reduces inflammatory cell infiltration and collagen deposition, delaying the occurrence of capsular contracture, although the underlying mechanism needs further indication.

\section{Conclusion}

We report that microgroove-patterned silicone rubber surface with C-ion implantation can provide enhanced biocompatibility and reduce the incidence of capsule contracture. These effects are mainly realized by changes in the topography of the material surface and improvements in its physical and chemical properties. Because this approach is simple and cheap to implement, we believe that it may have good prospects for clinical application in the future.

\section{Acknowledgment}

This work was supported by the military youth training program (Grant No 13QNP115). 


\section{Disclosure}

The authors report no conflicts of interest in this work.

\section{References}

1. Leszczynski R, Gumula T, Stodolak-Zych E, et al. Histopathological evaluation of a hydrophobic terpolymer (PTFE-PVD-PP) as an implant material for nonpenetrating very deep sclerectomy. Invest Ophthalmol Vis Sci. 2015;56(9):5203-5209.

2. Trumpy IG, Roald B, Lyberg T. Soft tissue response to polytetrafluoroethylene and silicone rubber in humans: morphological and immunohistochemical observations. Scand J Plast Reconstr Surg Hand Surg. 1997;31(4):295-301.

3. Xu R, Luo G, Xia H, et al. Novel bilayer wound dressing composed of silicone rubber with particular micropores enhanced wound re-epithelialization and contraction. Biomaterials. 2015;40:1-11.

4. Matsuoka M, Akasaka T, Hashimoto T, Totsuka Y, Watari F. Improvement in cell proliferation on silicone rubber by carbon nanotube coating. Biomed Mater Eng. 2009;19(2-3):155-162.

5. Evans SL, Gregson PJ. The effect of a plasma-sprayed hydroxyapatite coating on the fatigue properties of Ti-6al-4v. Mater Lett. 1993; 16(5):270-274.

6. Rahman CV, Ben-David D, Dhillon A, et al. Controlled release of BMP-2 from a sintered polymer scaffold enhances bone repair in a mouse calvarial defect model. J Tissue Eng Regen Med. 2014;8(1): 59-66.

7. Ling $\mathrm{T}$, Lin $\mathrm{J}, \mathrm{Tu} \mathrm{J}$, et al. Mineralized collagen coatings formed by electrochemical deposition. J Mater Sci Mater Med. 2013;24(12): 2709-2718.

8. Liu P, Chen Q, Yuan B, et al. Facile surface modification of silicone rubber with zwitterionic polymers for improving blood compatibility. Mater Sci Eng C Mater Biol Appl. 2013;33(7):3865-3874.

9. Wang SL, Shi XH, Yang Z, et al. Osteopontin (OPN) is an important protein to mediate improvements in the biocompatibility of $\mathrm{C}$ ionimplanted silicone rubber. PLoS One. 2014;9(6):e98320.

10. Zhou X, Chen X, Mao TC, et al. Carbon ion implantation: a good method to enhance the biocompatibility of silicone rubber. Plast Reconstr Surg. 2016;137(4):690e-699e.

11. Knight MAF, Evans GRD. Tissue engineering: progress and challenges. Plast Reconstr Surg. 2004;114(2):26E-37E.

12. Discher DE, Janmey P, Wang YL. Tissue cells feel and respond to the stiffness of their substrate. Science. 2005;310(5751):1139e43.
13. Khetan S, Burdick JA. Patterning network structure to spatially control cellular remodeling and stem cell fate within 3-dimensional hydrogels. Biomaterials. 2010;31(32):8228e34.

14. Chen S, Jones JA, Xu Y, Low HY, Anderson JM, Leong KW. Characterization of topographical effects on macrophage behavior in a foreign body response model. Biomaterials. 2010;31(13):3479e91.

15. Filova E, Bullett NA, Bacáková L, et al. Regionally-selective cell colonization of micropatterned surfaces prepared by plasma polymerization of acrylic acid and 1,7-octadiene. Physiol Res. 2009;58(5):669-684.

16. Meyle J, Wolburg H, von Recum AF. Surface micromorphology and cellular interactions. J Biomater Appl. 1993;7(4):362-374.

17. Yan C, Sun JG, Ding JD. Critical areas of cell adhesion on micropatterned surfaces. Biomaterials. 2011;32(16):3931e8.

18. Dolatshahi-Pirouz A, Jensen T, Kraft DC, et al. Fibronectin adsorption, cell adhesion, and proliferation on nanostructured tantalum surfaces. ACS Nano. 2010;4(5):2874-2882.

19. Stevens MM, George JH. Exploring and engineering the cell surface interface. Science. 2005;310(5751):1135-1138.

20. Ishikawa J. Negative-ion source applications. Rev Sci Instrum. 2008; 79(2 pt 2):02C506.

21. Elloumi Hannachi I, Itoga K, Kumashiro Y, Kobayashi J, Yamato M, Okano T. Fabrication of transferable micropatterned-co-cultured cell sheets with microcontact printing. Biomaterials. 2009;30(29): $5427-5432$.

22. Tsuji H, Sommani P, Kitamura T, et al. Nerve-cell attachment properties of polystyrene and silicone rubber modified by carbon negative-ion implantation. Surf Coat Technol. 2007;201(19-20):8123-8126.

23. Tsuji H, Sommani P, Hattori M, et al. Adhesion patterning of mesenchymal stem cells on polystyrene surface by carbon negative-ion implantation and neuron differentiation on the position. Nucl Instrum Methods Phys Res B. 2008;266(12-13):3067-3070.

24. Kjoller K, Holmich LR, Jacobsen PH, et al. Capsular contracture after cosmetic breast implant surgery in Denmark. Ann Plast Surg. 2001; 47(4):359-366.

25. Barnsley GP, Sigurdson LJ, Barnsley SE. Textured surface breast implants in the prevention of capsular contracture among breast augmentation patients: a meta-analysis of randomized controlled trials. Plast Reconstr Surg. 2006;117(7):2182-2190.

26. Madekurozwa MC. Post-hatch changes in the immunoexpression of desmin, smooth muscle actin and vimentin in the testicular capsule and interstitial tissue of the Japanese quail (Coturnix coturnix japonica). Anat Histol Embryol. 2013;42(5):369-378.
International Journal of Nanomedicine

\section{Publish your work in this journal}

The International Journal of Nanomedicine is an international, peerreviewed journal focusing on the application of nanotechnology in diagnostics, therapeutics, and drug delivery systems throughout the biomedical field. This journal is indexed on PubMed Central, MedLine, CAS, SciSearch ${ }^{\circledR}$, Current Contents ${ }^{\circledR} /$ Clinical Medicine,

\section{Dovepress}

Journal Citation Reports/Science Edition, EMBase, Scopus and the Elsevier Bibliographic databases. The manuscript management system is completely online and includes a very quick and fair peer-review system, which is all easy to use. Visit http://www.dovepress.com/ testimonials.php to read real quotes from published authors. 